\title{
EchoGéo
}

39 | 2017

Ressources urbaines (1)

\section{La fabrique de la géographie urbaine critique et radicale}

Cécile Gintrac

\section{(2) OpenEdition \\ 12 Journals}

\section{Electronic version}

URL: https://journals.openedition.org/echogeo/14901

DOI: 10.4000/echogeo.14901

ISSN: 1963-1197

\section{Publisher}

Pôle de recherche pour l'organisation et la diffusion de l'information géographique (CNRS UMR 8586)

\section{Electronic reference}

Cécile Gintrac, "La fabrique de la géographie urbaine critique et radicale", EchoGéo [Online], 39 | 2017, Online since 28 March 2017, connection on 10 August 2021. URL: http://journals.openedition.org/ echogeo/14901; DOI: https://doi.org/10.4000/echogeo.14901

This text was automatically generated on 10 August 2021

EchoGéo est mis à disposition selon les termes de la licence Creative Commons Attribution - Pas d'Utilisation Commerciale - Pas de Modification 4.0 International (CC BY-NC-ND) 


\title{
La fabrique de la géographie urbaine critique et radicale
}

\author{
Cécile Gintrac
}

1 En France, les années 2010, 2011 et 2012 ont été marquées par la multiplication de traductions (voir Harvey, 2010 et 2012) et de publications (voir Garnier J.-P., 2010 ; Clerval A., 2010 ; le dossier «Géographies critiques » de la revue Carnets de géographes (2012) autour de la géographie urbaine critique et radicale). En 2011, lorsque la revue Carnets de géographes lance son appel à articles sur les " géographies critiques ", elle propose de les définir comme "l'ensemble des approches construites autour d'une remise en question des catégories analytiques classiques pour lire le monde, articulées à une volonté de peser sur les évolutions politiques et sociales des sociétés étudiées. Dans les géographies anglo-saxonnes, les approches critiques sont généralement associées à une posture radicale, critique du capitalisme et du néolibéralisme comme forces de structuration dominantes, et des courants d'oppositions critiques afférents (postcultural studies ou subaltern studies par exemple) »(Calbérac et Morange, 2011).

2 L'approche radicale, plus explicitement liée aux approches marxistes, dénonce principalement la fabrique capitaliste de l'espace urbain. Néanmoins, «l'un et l'autre ont en commun de se positionner contre des théories hégémoniques issues du néolibéralisme (compétitivité, classe créative, régénération urbaine) et contre leur mise en pratique dans le cadre des politiques urbaines métropolitaines » (Gintrac et Giroud, 2014).

Du Brésil au Japon en passant par l'Espagne ou encore l'Afrique, plusieurs groupes de géographes se revendiquent actuellement de l'approche critique et radicale de la ville. Plutôt que de partir des définitions générales, il semblait utile de chercher à comprendre ce que signifiait la géographie critique et radicale pour ces groupes, ce que cela impliquait concrètement en terme de fonctionnement collectif, de pratiques de recherche, mais aussi de rapport à l'université et à l'engagement.

4 Pour traiter de cette "science en train de se faire ", il a paru nécessaire de puiser dans les travaux de sociologie des sciences et plus largement des science studies (STS). Cette approche permet de ne pas restreindre l'analyse aux seuls contenus de la recherche, 
comme le fait souvent l'épistémologie, mais de s'intéresser également aux pratiques des chercheur.se.s. La science n'est pas alors un "donné » figé dans des textes, elle est construite et sans cesse en mouvement. J'ai donc choisi d'étudier cette «fabrique », en suivant trois groupes contemporains: le GESP (Grupo de Estudos sobre São Paulo) au Brésil, Kritische Geographie Berlin en Allemagne et le réseau international INURA (International Network for Urban Research and Action) qui s'identifient explicitement à la géographie critique urbaine. Malgré leur éloignement géographique et leurs différences culturelles et scientifiques, ils ont en commun une approche critique de la ville.

5 Ainsi, la comparaison des pratiques et des savoirs produits permet-elle de dégager les traits concordants d'une géographie critique urbaine et de définir plus nettement ce qu'elle recouvre? Les trois groupes relativement périphériques par rapport aux lieux les plus visibles de la production scientifique sont-ils en décalage par rapport à des géographies critiques anglophones?

$6 \quad$ L'étude de la géographie critique urbaine par le prisme des science studies repose sur quelques postulats méthodologiques qu'il conviendra d'exposer dans un premier temps. Les trois groupes retenus seront ensuite présentés. Puis dans un dernier temps, il s'agira de proposer une définition de la géographie critique urbaine en recoupant les points communs aux trois groupes.

\section{L'apport des sciences studies pour étudier la science en action}

7 Les sciences studies peuvent offrir une vision inédite de la géographie critique urbaine contemporaine qui diffère de la seule histoire des idées.

8 À partir des années 1970, les sciences studies ont fourni de précieux outils pour reconsidérer l'étude de la science, non pas seulement comme une analyse des contenus scientifiques mais comme activité sociale. L'appellation désigne un vaste ensemble de travaux interdisciplinaires qui ont pour point commun d'envisager la science « comme une institution - et plus seulement comme un savoir. Elle est abordée comme un ensemble de pratiques et de faire, au laboratoire ou sur le terrain - et plus seulement comme un ensemble conceptuel» (Pestre, 2006, p. 3). On peut considérer que ce sont les travaux de David Bloor et de Harry Collins qui inaugurent les sciences studies. En 1976, le premier fonde une nouvelle démarche, le "programme fort » qui tend à désacraliser l'activité scientifique et à rendre possible la description et l'analyse d'une réalité conçue avant tout comme une activité sociale. C'est ensuite, Bruno Latour et le sociologue britannique Steve Woolgar qui ont proposé, dans Laboratory Life paru en 1979 (La vie de laboratoire comme il fut traduit ultérieurement en français), de s'intéresser moins aux connaissances elles-mêmes qu'à leur élaboration quotidienne, dans une « description anthropologique au ras du sol» (Pestre, 2006, p. 44)

Les sciences studies consistent «à parler de pratique scientifique et à proposer de ce fait un exposé plus réaliste de la science-en-train-de-se-faire, l'enracinant fermement dans les laboratoires, les expériences et les groupes de collègues» (Latour, 2007, p. 23). Autrement dit, pour comprendre la science, les contenus ne sauraient être étudiés en les déconnectant de l'observation des pratiques, quotidiennes ou plus exceptionnelles, des chercheurs qui les produisent ainsi que des conditions de production des faits 
scientifiques. Il s'agit bien de ne pas se limiter à une épistémologie internaliste, analysant uniquement des contenus, mais bien de les articuler à une perspective externaliste de la science, comme analyse des pratiques, voire à une " anthropologie ", pour reprendre les termes de Latour, de l'activité scientifique.

Ainsi, elles autorisent une description matérialiste de l'activité scientifique. Néanmoins pour éviter de restreindre l'analyse à la seule échelle du laboratoire, il faut, avec Bourdieu, s'intéresser à «la position du laboratoire dans une structure " globale (Bourdieu, 2001, p. 75), ce qui suppose d'intégrer à l'étude les dispositifs de gouvernement des sciences (législations ou financements par exemple), mais aussi aux rapports de force entre les laboratoires, les universités ou les centres de recherche.

11 Reste à savoir, comment, méthodologiquement, conduire cette étude et surtout à quel niveau d'observation la conduire.

\section{Passer des courants de pensée aux communautés épistémiques}

12 À bien des égards, le groupe de recherche est apparu comme le niveau le plus pertinent. Il renvoie à la notion de "communauté épistémique ", qui s'inscrit dans la lignée des sciences studies (Haas, 1992), et qualifie un groupe uni par des valeurs et une approche scientifique communes. Se constituer en tant que groupe suppose de se donner un nom et définir des valeurs, qui créent une forte unité interne.

Il s'agit d'étudier des groupes auto-constitués. Ainsi, "au lieu de supposer que les communautés épistémiques existent a priori, nous nous devons d'examiner comment elles naissent, comment elles sont fabriquées et matérialisées, ce qui nécessite l'exploration des pratiques, des objets, des métaphores, des instruments et des discours qui produisent et maintiennent ces collectivités » (Meyer et Molyneux-Hodgson, 2011, p. 149).

14 Dans cette perspective, la géographie critique ne serait alors que la somme des communautés épistémiques qui s'en revendiquent, susceptibles de former un réseau plus ou moins cohérent

15 Le concept de communauté épistémique et la méthode d'analyse qu'il impose résolvent la plupart des problèmes méthodologiques posés par l'étude d'un vaste courant de pensée :

- les modalités d'(auto)-organisation du groupe, qui font le plus souvent l'objet de délibérations et de débats collectifs, sont représentatives des valeurs portées par les groupes ;

- la démarche inductive n'impose pas de choisir des limites temporelles ou géographiques artificielles : chaque groupe se déploie dans le temps, à travers une histoire particulière, et dans l'espace, à travers le réseau constitué par ses membres et les liens tissés avec d'autres groupes et institutions ;

- le groupe permet aussi de ne pas se limiter aux grands noms d'un courant et de sortir des seuls récits individuels. Il permet de s'intéresser à tous les membres du groupe, quels que soient leur statut.

La communauté épistémique est donc une notion opérante et correspond particulièrement bien à l'étude des approches critiques: elle permet d'envisager le 
collectif plutôt que l'individu, l'auto-organisation plutôt que l'institution, les pratiques concrètes plutôt que les ouvrages canoniques.

\section{Trois groupes se réclamant de la géographie urbaine critique}

17 Quoique contemporains, les groupes sélectionnés évoluent dans des contextes linguistiques et institutionnels très variés.

18 Le GESP est implanté à l'Université de Sao Paulo, dans une métropole dite du "Sud ». Kritische Geographie Berlin est situé dans la capitale d'un grand pays industrialisé, mais ses activités se font hors des locaux de l'université (ancien squat, bars, locaux associatifs). INURA est un réseau international dont les membres viennent du monde entier, mais il est administrativement domicilié à Zürich et reste, dans sa composition, essentiellement européen. Il existe réellement lorsque ses membres se rencontrent lors des conférences, dans les hôtels, les lieux de conférences, les excursions sur le terrain. Deux de ces groupes ont une implantation urbaine et métropolitaine, tandis qu'INURA se présente comme un réseau international et itinérant, en ce qu'il organise des conférences annuelles dont le lieu d'accueil change chaque année. Le nombre des membres au sein de Kritische Geographie Berlin et du GESP reste modeste (entre 15 et 25), tandis qu'INURA compte une centaine de membres.

Les trois groupes choisis sont dans une certaine mesure "périphériques", si l'on considère comme centraux les principaux lieux de publication et de diffusion des savoirs en géographie urbaine et études urbaines dont les revues de référence et les principales conférences sont anglophones voire anglo-américaines. À l'exception d'INURA, réseau au sein duquel l'anglais est la principale langue de communication (mais rarement la langue maternelle des membres), les groupes sélectionnés privilégient les publications dans leur propre langue (portugais ou allemand) et une diffusion avant tout locale ou nationale. Même si cela est affirmé comme un choix, cette hiérarchisation linguistique est aussi le fait d'une certaine marginalisation. Au début des années 2000, la géographie critique s'est précisément intéressée à la géographie du savoir en montrant comment l'anglais est devenue la lingua franca de la recherche et de la géographie critique urbaine également. « Les autres langues, et ceux qui les parlent, semblent exclues, opprimées et contraintes à se soumettre à l'hégémonie anglophone » (Best, 2009, p. 83).

20 La sélection de ces trois groupes relève donc d'un choix personnel assumé de ne pas mettre au cœur de l'analyse les auteurs déjà publiés et les plus commentés de la géographie critique. Cela s'inscrit également dans l'objectif de mieux faire connaître la géographie critique urbaine, dans sa diversité.

21 Une série d'enquêtes de terrain a été menée entre 2012 et 2015, auprès des trois groupes de recherche, sur le terrain multi-situé des lieux pratiqués (universités, centres associatifs,...). Elle s'appuie sur l'analyse des interviews, des conférences, réunions, productions écrites, bibliographies, pratiques des trois groupes étudiés, afin d'étudier la géographie critique urbaine en action, de Berlin à Brasilia ou Lisbonne... 


\section{Le GESP (Grupo de Estudos sobre São Paulo) un groupe de recherche marxiste-lefebvrien}

Il peut paraître paradoxal qu'un groupe de recherche qui se présente comme radical soit rattaché à la plus puissante université d'Amérique latine. Fondée en 1934, l'USP (Universidade de São Paulo) est une université publique, qui dépend de l'État fédéré de Sao Paulo et regroupe environ 80000 étudiants. Le GESP s'est développé au sein du département de géographie, lui-même rattaché à la Faculdade de Filosofia, Letras $e$ Ciências Humanas. La FFLCH combine l'ambition de former l'élite du pays avec « un esprit de critique et de contestation » que le site de la faculté lui-même se plaît à mettre en avant. Cela explique que le GESP et le LABUR (Laboratório de Geografia Urbana), au sein duquel il a été créé, jouissent d'une grande autonomie, tant pour la recherche que pour l'enseignement.

Ce groupe de recherche, qui compte une vingtaine de membres en 2015, s'est constitué de manière autonome, sans financement ou hors des structures du laboratoire et du département. Depuis 2001, il organise, plus ou moins régulièrement, des groupes de lecture autour de la production capitaliste de l'espace urbain. Cette pratique des lectures et discussions collectives est centrale: elle fonde la réflexion théorique du GESP. Les membres du groupe cherchent ensuite à s'appuyer sur la diversité de leurs recherches de terrains pour accéder à la «totalité métropolitaine » de Sao Paulo. Cela suppose un travail théorique approfondi et des discussions à partir d'ouvrages : les œuvres de Lefebvre, bien sûr, mais aussi des textes contemporains. Ce travail de réflexion, collectif, n'est pas né avec le groupe, en 2001. Il est le fruit d'une longue tradition de la FFLCH, comme en témoigne Ana Fani Alessandri Carlos :

Pendant 18 ans, tous les vendredis matin, 5 mois par an, de 9 heures à 13 heures, on a lu et étudié Marx dans le groupe créé par le sociologue Martins en 1975. On lisait chacun chez soi et on discutait pendant 3 heures ou 3 heures et demie. On a lu tout le Capital, les Grundrisse, l'Idéologie allemande... on a lu presque tout ce qu'on pouvait lire de Marx. Puis le professeur Martins a proposé de lire l'œuvre d'un intellectuel dans le prolongement de la pensée de Karl Marx : il s'agissait d'Henri Lefebvre... À partir de 1989, avec ce même style de travail, mais puisque nous étions géographes, on a commencé à lire le Droit à la Ville, la Production de l'espace, la Révolution urbaine... (Carlos, entretien en français, 2011).

Ce n'est pas seulement le Droit à la ville, référence majeure de la géographie brésilienne, qui est mobilisé par le GESP, mais tout l'éventail des concepts lefebvriens (production, vie quotidienne, espace-temps) qui constitue le noyau théorique du GESP (Carlos, 2015).

Le groupe compte aujourd'hui, parmi sa vingtaine de membres, une moitié de professeurs et l'autre d'étudiants. Parmi les professeurs, on distingue trois figures de la géographie radicale brésilienne: Ana Fani Alessandri Carlos, Ariovaldo Umbelino de Oliveira et Arlete Moysés Rodrigues. Les femmes y sont majoritaires, y compris parmi les professeurs (neuf sont des femmes). La recherche du GESP se veut collective, au-delà des statuts universitaires variés de ses membres et le groupe n'a pas hésité à exprimer publiquement des critiques du productivisme universitaire (Carlos 2012 ; Cruz, 2012).

Pour autant, le GESP jouit d'une grande légitimité scientifique au Brésil à la fois du fait de son implantation à l'USP mais aussi parce ses membres ont contribué à créer des espaces et des supports reconnus : revues, collections, conférences. C'est le cas par exemple de la principale conférence de géographie urbaine SIMPURB (Simpósio Nacional 
de Geografia Urbana), créée en 1989 à l'initiative d'Ana Fani Alessandri Carlos et qui regroupe, tous les deux ans, des centaines de chercheurs.

Le GESP milite explicitement pour " une autre ville » en soutenant la «lutte pour le droit à la ville» (GESP, 2014). Plusieurs membres accompagnent les luttes urbaines, notamment des favelas. Ils ont fondé un sous-groupe Resistências qui travaille notamment sur les résistances aux expulsions. Cependant, le «terrain » d'action du GESP se situe avant tout à l'université. Ainsi, ses membres ne se sont jamais désintéressés de la pédagogie, en contribuant par exemple à la formation continue des enseignants de l'État de Sao Paulo. Ana Fani Alessandri Carlos et Arlete Moysés Rodrigues ont publié des ouvrages didactiques largement diffusés et réédités à plusieurs reprises: A Cidade, A geografia na sala da aula (Carlos, 2006) et Moradia nas Cidades Brasileiras ${ }^{2}$ (Rodrigues, 1988). Ces petits ouvrages sont massivement utilisés par les enseignants du secondaire.

Le GESP se présente donc comme un groupe de géographie critique et radical reconnu au sein de l'université brésilienne qui place au cœur de sa démarche la théorie lefebvrienne. À ce titre, la radicalité du GESP se place avant tout sur le plan théorique mais elle n'en a pas moins une réelle influence sociale grâce aux relais que sont les géographies universitaire et scolaire.

\section{INURA (International Network for Urban Research and Action) : un altermondialisme urbain}

Ce réseau a été créé en mai 1991, un an après que l'idée a émergé lors d'une rencontre à Zürich, à l'initiative de jeunes géographes zurichois qui cherchent à fonder un réseau transdisciplinaire, au-delà des murs de l'université. INURA s'est d'emblée pensé comme un groupe ouvert aux artistes, aux militants politiques autant qu'aux chercheurs (principe 10 de la déclaration d'intention).

Dès 1991, la décision est aussi prise de tenir une conférence chaque année dans un lieu différent, européen dans un premier temps. Ouverte à tous, celle-ci est organisée et conçue par une équipe de membres volontaires. En 2014 par exemple, à Belgrade, c'est un collectif Ministarstvo Prostora (Ministère de l'espace) qui en avait la charge. Ce groupe militant qui réunit des architectes, des chercheurs, des designers, des artistes n'est pas lié à l'université. La géographie même de la conférence de 2014, - dispersées dans de nombreux lieux, tantôt universitaires, tantôt municipaux, culturels, voire militants, souligne le fait que la rencontre n'est pas une conférence universitaire classique.

Son originalité tient aussi aux larges plages de visites urbaines. Les conférences d'INURA offrent donc à ses membres l'opportunité de découvrir, dans chaque ville où elles se tiennent, les mouvements urbains qui s'y sont développés. À Lisbonne comme à Belgrade, des tables rondes donnaient la parole à des associations et aux ONG. Nombreux d'ailleurs sont les membres d'INURA qui ont milité dans leur jeunesse ou militent encore dans le cadre de luttes urbaines. Rencontrer les groupes mobilisés compte tout autant - voire davantage - pour eux que d'accumuler des connaissances scientifiques. Le slogan "un autre monde urbain est possible $»^{3}$ ou la participation au Forum social de Porto Alegre en 2005 viennent rappeler qu'INURA se donne pour objectif de transformer la réalité urbaine, dans une conception proche du mouvement altermondialiste né dans les années 1990. 
Illustration 1 - Rencontre avec les associations d'habitants de Vale da Amoreira le 19 juin 2013

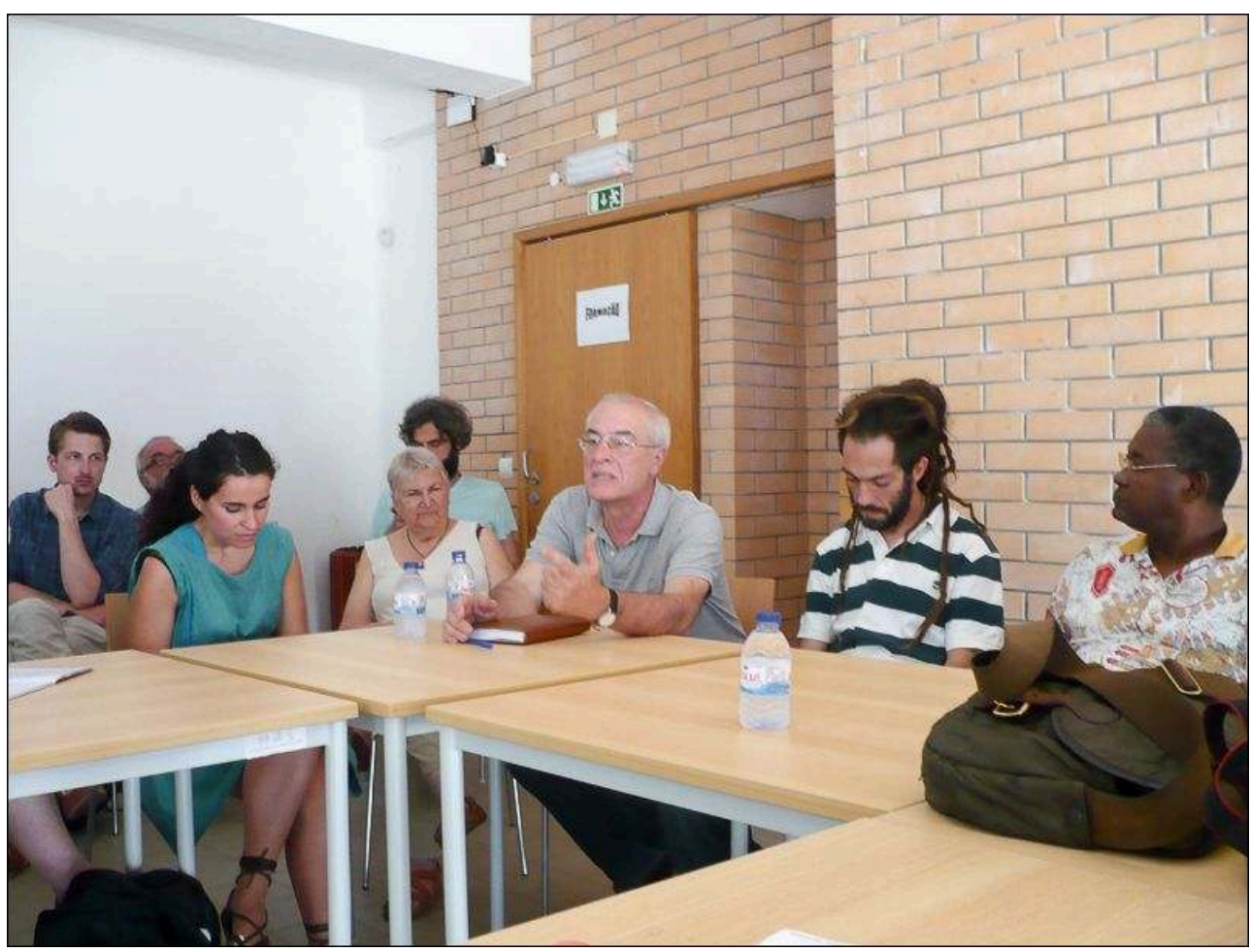

Le chercheur et militant António Guterres traduisait au fur et à mesure les propos des habitants. Les membres d'INURA écoutent puis posent des questions avant de visiter le quartier.

Auteur : C. Gintrac.

INURA entend du reste s'insérer dans les débats des villes qui l'accueillent et peser, dans la mesure du possible, dans les décisions d'aménagement en cours. À Lisbonne, il s'agissait de s'opposer à l'expulsion d'habitants ainsi qu'à la destruction des jardins du quartier Santa Filomena, qu'une partie des participants avaient découvert lors d'une visite organisée. À l'unanimité, les participants décidèrent, pendant la retraite qui suivait, de rédiger une lettre à la municipalité pour dénoncer cette expulsion. À Belgrade, l'équipe organisatrice, impliquée dans la lutte contre le projet pharaonique de reconquête du front d'eau (Belgrade on Water), avait pris le soin de programmer un événement public dans un cinéma de la capitale serbe.

Chaque conférence est suivie d'une "retraite » (retreat), c'est-à-dire d'une réunion de plusieurs jours, consacrée à la réflexion collective sur le réseau, dont les membres sont géographiquement dispersés. C'est un moment réflexif essentiel où des questionnements variés émergent sur les pratiques et l'organisation même d'INURA.

La convivialité et les liens d'amitié qui se sont tissés font partie des raisons du succès et de la fidélité des membres. Les principes d'auto-organisation et d'horizontalité qui structurent le groupe se matérialisent concrètement par les publications collectives. C'est le cas des ouvrages suivants : Possible Urban World Urban: Strategies at the End of the 20th Century et The Contested Metropolis Six Cities at the Beginning of the 21st Century, (INURA, 1999; 2004). Dans la continuité de ces travaux, INURA s'est lancé depuis 2008 dans un vaste projet commun de recherche autour du "New Metropolitan Mainstream » (NMM), ce "nouveau sens commun métropolitain » qui uniformise les paysages et la vie urbaine. Cette recherche collective et internationale se présente sous 
la forme d'une cartographie des métropoles réalisée par les membres et a donné lieu à plusieurs expositions.

Si la création d'INURA revient essentiellement à des géographes, ceux-ci, dès l'origine, tenaient à ne pas limiter le réseau aux simples frontières disciplinaires. En ce sens, INURA est véritablement un réseau d'études urbaines (urban studies), dont le point de départ est moins l'approche disciplinaire que l'objet urbain. Les professionnels de l'aménagement, urbanistes, architectes, ingénieurs urbains constituent à ce titre une part non négligeable des membres.

Entre 1991 et aujourd'hui le réseau, qui est aussi une association, a gagné des membres pour atteindre 93 adhérents et s'est étendu géographiquement dans le monde (même si la majorité des membres restent européens). La liste de diffusion comptait plus de 350 abonnés en juin 2014. INURA s'est aussi doté d'une structure stable et organisée, matérialisée par l'INURA Common Office. Basé à Zurich, ce bureau, créé par Richard Wolff et Philippe Klaus en 1999, permet de faire fonctionner l'association au quotidien.

INURA a donc réussi à créer réseau international sur la longue durée. Cette exceptionnelle pérennité tient aux solides liens tissés au fur et à mesure des années mais aussi à l'originalité des conférences/retraites. Ce réseau né au début des années 1990 fait vivre une forme d'altermondialisme urbain dont il se revendique.

\section{Kritische Geographie Berlin, la géographie critique hors les murs de l'université}

C'est un groupe à l'image de la vie sociale berlinoise : précaire, auto-organisé, ouvert mais il participe, depuis sa création, à animer la géographie critique allemande.

Les réunions mensuelles de Kritische Geographie Berlin se tiennent à Kreuzberg, dans les locaux de l'association Babylonia. Cet ancien squat est devenu l'adresse officielle de l'association du même nom créée en 2012. Le choix de ce lieu indépendant est en cohérence avec ses principes : «Kritische Geographie Berlin est un collectif indépendant de gauche, qui n'est lié à aucun parti, université ou institution publique » (Über uns, site internet).

Le vendredi 18 juillet 2014 par exemple, neuf membres du groupe s'installent sur les bancs et se retrouvent pour s'informer et faire le bilan des projets en cours. Les différents projets du groupe (participation aux conférences et festivals, organisation d'ateliers, réalisation d'un atlas militant, organisation des «excursions »,..) sont discutés. La parole y circule librement. 
Illustration 2 - L'entrée de Babylonia, vendredi 18 juillet 2014, avant la réunion de Kritische Geographie Berlin

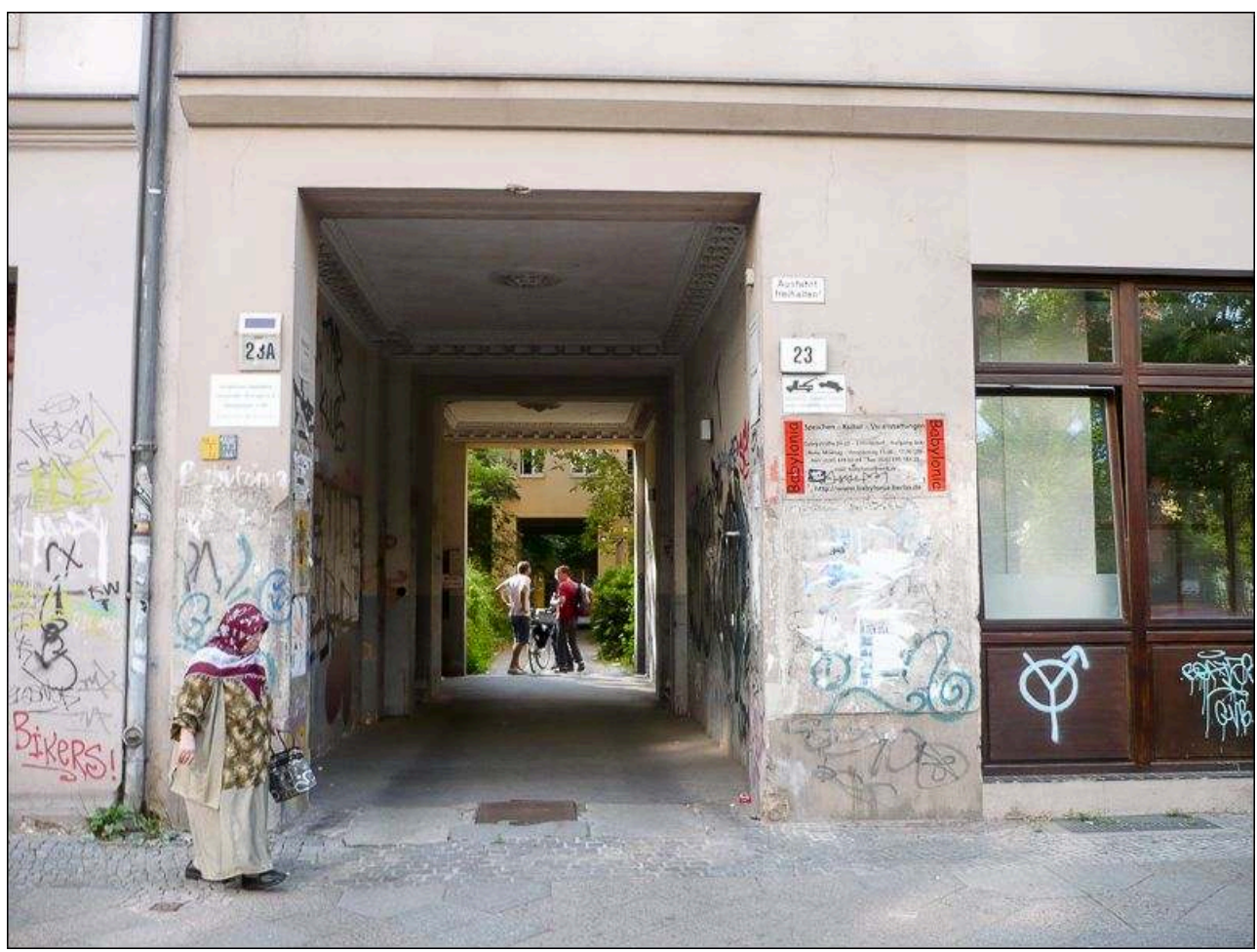

Auteur : C. Gintrac.

Les débuts du groupe remontent à l'année 2000 au moment où plusieurs étudiants, qui ont découvert la géographie radicale lors de séjours dans les universités anglophones, s'organisent en groupe de lecture autonome. À cette période, la géographie allemande apparaît comme une discipline conservatrice. Jusqu'en 2005, ces réunions " consistaient surtout à s'auto-organiser en tant que groupe, à auto-organiser notre construction du savoir et à trouver ce que nous voulions faire avec notre savoir " (entretien avec Th. Bürk). Cette préoccupation est entrée en résonnance avec d'autres initiatives conduites à la même période, notamment les "Doreen Massey week-ends », une série de conférences organisées par un groupe de géographes féministes entre 1999 et 2004. Cette série d'événements a donné une légitimité nouvelle à la géographie féministe allemande (B.A.S.S.D.A, 2006), à la faveur de l'internationalisation de la géographie allemande (Belina et al., 2009).

42 Malgré cette nouvelle dynamique, les activités du groupe s'essoufflent à partir de 2008. La plupart des membres achèvent à cette période leur thèse ou leurs contrats de Wissenschaftlicher Mitarbeiter et quittent Berlin. La véritable renaissance du groupe intervient donc en 2010. Le principe d'une réunion mensuelle ouverte est adopté. Cette mutation transforme le groupe de lecture, basé essentiellement sur des relations amicales, en un véritable collectif plus ouvert, devenu une association en 2012.

Ce que propose Kritische Geographie Berlin, c'est donc de faire de la géographie sans que le statut, ni le rang social ou le niveau d'études n'interviennent dans cette pratique. L'intense mouvement de précarisation explique la nécessité de trouver un lieu hors université pour faire ou diffuser de la géographie critique, avec ou sans poste, avec ou sans contrat de recherche. Kritische Geographie Berlin prend ouvertement position contre 
cette logique de précarisation de la recherche et la commercialisation du savoir. En 2012 par exemple, elle a lancé, avec d'autres chercheurs et collectifs, un appel au boycott de certains contrats de recrutement ${ }^{4}$ particulièrement défavorables aux chercheurs. Il s'agit donc ici de peser sur les conditions de production de la recherche et pas seulement sur le contenu de celle-ci.

L'objectif du groupe est donc moins de produire des savoirs que de s'intéresser aux modalités de sa transmission et de sa diffusion. Ainsi le groupe organise, à Babylonia, des ateliers ouverts à un plus large public et propose des excursions critiques dans Berlin, ouvertes à tous, et pensées comme une pratique alternative de la géographie. À titre d'exemple, le 19 juillet 2014, l'excursion intitulée «Un jour d'été à Postdam : contre-culture, l'esprit du passé et le droit à la ville" s'intéressait à la « disneylandisation » de cette ancienne ville de la RDA, située à $25 \mathrm{~km}$ de Berlin, connue pour son château et ancienne résidence royale de Frédéric Guillaume I ${ }^{\mathrm{er}}$. L'excursion, ouverte à tous, propose de dénoncer la réhabilitation très sélective du patrimoine, à travers un parcours de trois heures et demie.

Faire de la géographie critique, cela peut donc consister à observer son quartier, son espace quotidien, et à prendre la mesure des conflits qui s'y déroulent.

Sur le fond, Kritische Geographie Berlin s'attache à étudier les dynamiques qui modifient en profondeur la vie quotidienne berlinoise, notamment l'intense processus de gentrification à l'œuvre dans la capitale - par exemple dans les quartiers de l'est berlinois, Kreuzberg ou Prenzlauer Berg et la touristification, qui a fait, en très peu de temps, de Berlin la troisième ville touristique européenne après Londres et Paris (Kritische Geographie Berlin, 2014 ; Füller et Michel, 2014).

Berlin apparaît comme un lieu privilégié des mouvements locaux, indépendants, autoorganisés. Habiter à Berlin implique, pour la plupart des membres du groupe, la participation à de nombreux groupes militants.

Au final, la géographie critique de Kritische Geographie Berlin reflète indéniablement la richesse et l'intensité de la vie sociale et militante berlinoise et ne se conçoit pas sans elle.

\section{Redessiner la géographie critique urbaine}

Les points communs aux trois groupes dessinent, par recoupement, les lignes structurantes de la géographie critique urbaine.

\section{L'ancrage normatif des déclarations d'intention}

Les trois groupes affirment des valeurs communes, définies dans leur déclaration d'intention. Cela revient à assumer publiquement un travail réflexif qui permet d'exposer d'où ces groupes parlent. Pour Razmig Keucheyan, un des principaux critères "permettant de situer les pensées critiques dans l'espace conceptuel des sciences humaines est lié au rapport entre le descriptif et le normatif. Les pensées critiques tendent à problématiser, parfois mêmes à récuser, la distinction entre ces deux instances " (Keucheyan, 2013, p. 101). De prime abord, ce positionnement semble en opposition franche avec la conception idéale - et, à dire vrai, surtout idéalisée - de la neutralité axiologique (Wertfreiheit), telle qu'elle a été définie par Max Weber. Le terme a souvent 
été invoqué "pour justifier l'image d'un savant libre de passions partisanes " (Pinto, 2011, p. 109).

Pour surmonter cette apparente contradiction, on peut avancer l'idée que «ce que défend la critique, c'est la nécessité de fonder rationnellement ses appuis normatifs, c'est-à-dire de les affirmer et de les expliciter pour qu'ils ne soient plus des implicites s'exerçant en contrebande » (Granjon, 2015). Autrement dit, il y aurait dans la déclaration d'intention une certaine honnêteté intellectuelle à ne pas passer sous silence les valeurs qui animent les chercheurs.

Dans ces textes, les groupes formulent ainsi explicitement des aspirations (" empowerment ", « justice », " droit », " émancipation » selon les groupes) et ce contre quoi ils s'engagent (" racisme ", " inégalités », « discriminations » de classe, de race, ou de genre). En procédant ainsi, ils participent à donner leur définition de la géographie critique urbaine: une pratique scientifique ouvertement engagée, dénonçant les formes urbaines de la domination et souhaitant participer à l'avènement d'une ville plus juste. Concrètement, cela se traduit en grande partie par le choix de certains thèmes de recherche: étude des groupes dominés (habitants des favelas, migrants, expulsés selon les cas) et des processus de domination urbaine.

\section{Les fondements théoriques communs}

La géographie critique mobilise un corpus théorique de gauche. Les pensées de gauche ont pour fondement la volonté de transformer l'ordre existant, tandis que les pensées de droite sont profondément réalistes, en ce sens qu'elles accordent un privilège aux faits acquis, à l'ordre établi, à «la force des choses » (Terray, 2012). Lawrence Berg ne dit pas autre chose dans une des encyclopédies de géographie humaine quand il affirme que si les géographes critiques ne se réclament pas tous du marxisme, « ils s'identifient tous volontiers comme progressistes de gauche » (Berg, 2010, p. 619).

En partant des bibliographies et des auteurs cités par les groupes, on constate que quatre auteurs forment le socle théorique commun aux trois groupes: David Harvey, Neil Smith, Edward Soja et surtout Henri Lefebvre. Ces auteurs ont pour particularité de proposer des théories de l'espace. 
Illustration 3 - Références théoriques communes et références particulières aux trois groupes

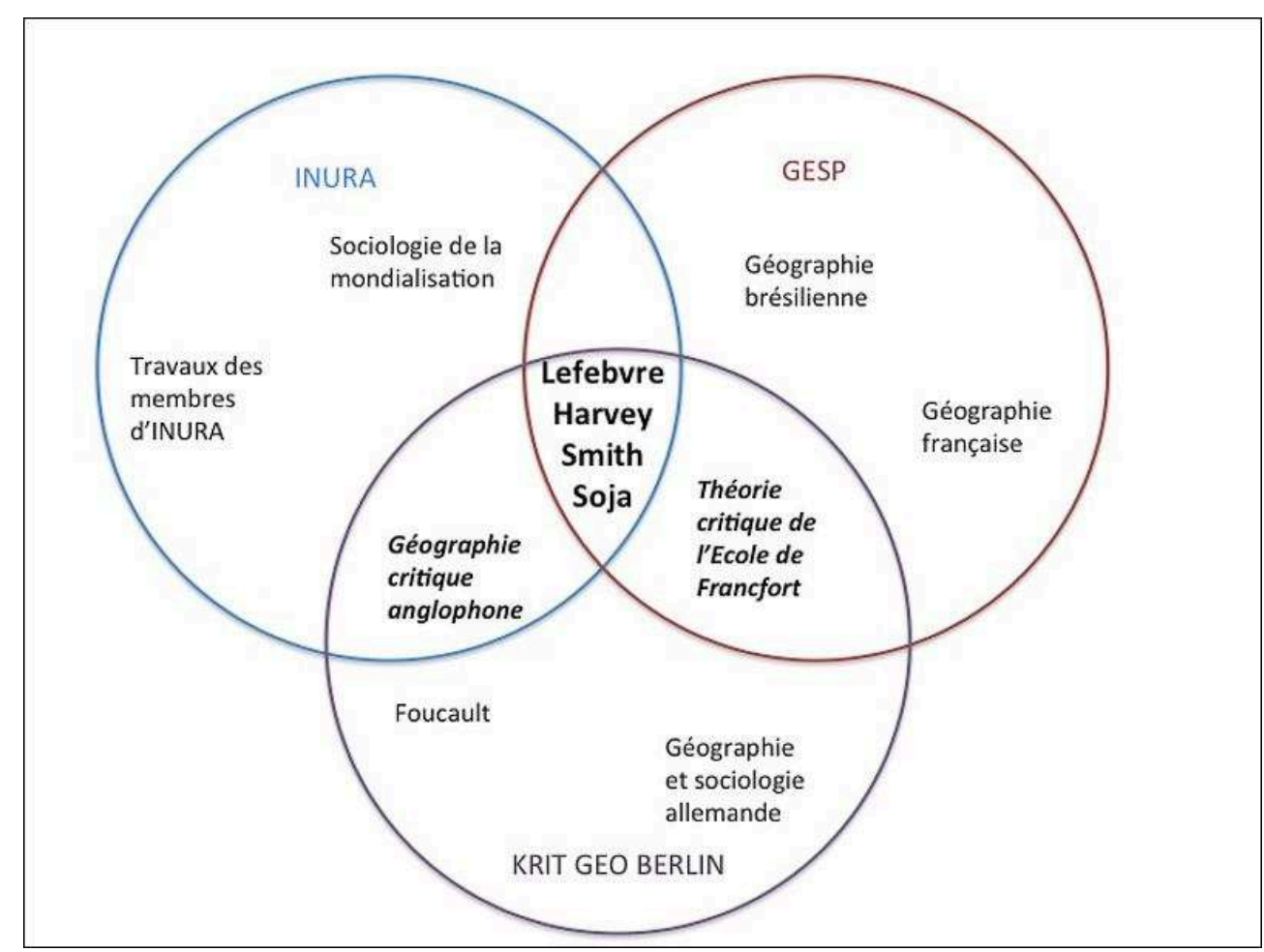

Schéma réalisé à partir des bibliographies des ouvrages : Handbuch Kritische Geographie (2014), Crise Urbana (2015) et The Contested Metropolis (2004)

(Bourdieu ou Foucault). Tous les penseurs ont pour point commun d'avoir pensé la domination sous des angles variés. Cela rejoint le constat de Lawrence Berg qui souligne dans l'entrée " géographie critique » d'une encyclopédie de la géographie que «les tenants de la géographie humaine critique s'appuient sur des approches théoriques telles que l'anarchisme, l'anticolonialisme, les théories critiques de la race (critical race theory), l'environnementalisme, le féminisme, le marxisme, la théorie nonreprésentationnelle, le post-marxisme, le postcolonialisme, le poststructuralisme, la psychanalyse, la théorie queer, le situationnisme, et le socialisme » (Berg, 2010, p. 616). L'importance du référentiel marxiste permet néanmoins d'affirmer que la plupart des groupes de géographie qui affirment produire une géographie critique produisent aussi une géographie radicale.

\section{Les savoirs produits}

La géographie urbaine critique, telle qu'elle est conçue par le GESP, Kritische Geographie Berlin ou INURA, possède une forte dimension théorique. Le plus souvent adossée à une théorie critique, elle vise une forme de «totalisation». Le sociologue Razmig Keucheyan, considère que le propre des pensées critiques est d'assumer « une certaine montée en 
généralité, elle ne se limite pas à un objet empirique circonscrit » (Keucheyan, 2013, p. 100).

Les savoirs produits par la géographie critique urbaine relèvent de l'analyse de la production capitaliste et/ou néolibérale de l'espace. Elle cherche à en comprendre les ressorts et les manifestations mais insiste sur les formes de résistance aux processus de dépossession et de négation de la vie quotidienne. Cela dégage un triptyque récurrent (production de l'espace/inégalités/résistances).

Un concept est repris unanimement par les trois groupes, celui de production de l'espace, inspiré de Lefebvre. Avec cette notion, il s'agit avant tout de comprendre les mécanismes à l'œuvre et les processus par lesquels le capital est produit et reproduit dans et par l'espace. Cette interprétation est directement tirée de La Production de l'espace: "chaque société (donc chaque mode de production avec les diversités qu'il englobe [...]) produit un espace, le sien » (2000 [1974], p. 40).

Mais l'on distingue des singularités d'un groupe à l'autre : la démarche comparatiste chez INURA, l'analyse des discours au sein de Kritische Geographie Berlin ou l'utilisation de la grille d'analyse lefebvrienne pour le GESP. Certains thèmes singuliers reflètent certaines dynamiques locales. À ce titre, le processus de «touristification » à Berlin ne peut faire sens à Sao Paulo et, inversement, la question de l'accès au foncier urbain pour les habitants des favelas ne permet pas de comprendre les transformations de la capitale allemande, ou les politiques d'austérité à Lisbonne sur lesquelles était centrée la conférence d'INURA en 2013.

\section{Transdisciplinarités}

Les trois groupes conçoivent la géographie critique urbaine comme une manière de dépasser la spécialisation des savoirs et la rupture entre monde universitaire, monde militant et monde social. Dans le Droit à la ville, Lefebvre oppose les «sciences parcellaires » à la « philosophie de la ville », qui envisage l'objet urbain dans sa totalité et «s'efforce de saisir le global» (p. 35). Puisque le savoir spécialisé ne permet pas de proposer une véritable critique propre à transformer la réalité, il n'est pas surprenant que la géographie critique urbaine ait largement recours à la philosophie (Marx, Foucault, De Certeau....) ou à la sociologie, ce que l'étude du corpus bibliographique a confirmé.

Il y a donc une visée transdiciplinaire que l'on retrouve au fondement même d'INURA ou encore dans le journal sublurban

Même si certains membres revendiquent spécifiquement une approche géographique, la géographie critique urbaine est de plus en plus intégrée aux études urbaines (critical urban studies), ce vaste champ auquel se rattachent de nombreux chercheurs. On notera que l'exigence intellectuelle de transdisciplinarité rejoint paradoxalement les injonctions de l'université contemporaine souvent qualifiée de néolibérale par les groupes étudiés.

\section{Contre le productivisme universitaire, pour des pratiques collectives}

Le constat de la néolibéralisation de l'université est largement partagé, dans les universités anglophones bien sûr, mais aussi dans les différents pays où exercent les géographes de nos groupes. Un nouveau «gouvernement des sciences»- pour 
reprendre l'expression de Bourdieu - se met en place, fondé sur la recherche croissante de la compétitivité entre universités et entre les chercheurs eux-mêmes. Les modes de production du savoir dans un monde universitaire de plus en plus concurrentiel ont été dénoncés à plusieurs reprises par les géographes critiques. En 2000, Neil Smith avait ainsi décrit l'université comme une "usine à saucisses " (sausage factory) et constaté que «la privatisation de l'université est un problème global et ses effets sont déjà présents parmi nous » (p. 331).

Leurs membres dénoncent régulièrement la manière dont le savoir est actuellement produit. Au Brésil comme en Allemagne, ils sont des observateurs attentifs de l'évolution de la recherche. Pour Kritische Geographie Berlin, la dégradation des conditions de la recherche est évidente: elle devient progressivement un bien marchand. Au Brésil, Rita de Cássia Cruz, chercheuse brésilienne et membre du GESP, qualifie la prestigieuse USP au sein de laquelle elle travaille d' " université néolibérale et néocoloniale » (Cruz, 2012). Concrètement, ces prises de position se traduisent par des pétitions, des textes d'interpellation ou même la démission de certaines instances pour dénoncer certaines pratiques.

Puisqu'il s'agit de transformer le monde, les savoirs produits se veulent autant que possible disponibles, gratuits et diffusés largement. Les collectifs s'efforcent de créer de nouveaux espaces, plus horizontaux et collectifs, de production du savoir (associations, groupes de recherche non financés, réseaux, publications chez des éditeurs indépendants). On retrouve chez les trois groupes «la volonté de sortir de 'l'enclos universitaire' » (Gintrac et Giroud, 2014)

\section{Un engagement politique à géométrie variable}

Les trois groupes ont une conception différente de l'engagement :

- le GESP, intégré à l'université, a surtout choisi de mener la lutte au sein de l'institution, dans une conception très bourdieusienne où le monde universitaire relève du rapport de force : un champ qu'il convient de ne pas abandonner. Cela est proche également de la conception du géographe américain Don Mitchell qui décrit son propre travail comme celui d'un « radical de bureau » (« deskbound radical»). Il considère que son rôle reste avant tout de produire un savoir, susceptible ensuite d'être mobilisé dans des luttes, plutôt que d'y participer activement (Mitchell, 2008). Cela tient à la sociologie universitaire brésilienne, où les chercheurs et étudiants viennent davantage d'une classe moyenne: l'engagement universitaire se pense parfois en soutien des mouvements de luttes urbains mais n'en est quasiment jamais issu ;

- INURA propose une vision nettement plus horizontale où les militants et les chercheurs sont placés au même niveau au sein de l'association. Dans ce cadre, le réseau est aussi un réseau d'expériences militantes localisées ;

- Kritische Geographie Berlin reste essentiellement un lieu d'échange de savoirs et de projets "para-universitaires", mais le groupe présente de fortes interactions avec les réseaux militants locaux, en lien avec l'histoire et la sociologie militante de Berlin.

68 Ces spécificités liées au groupe n'empêchent pas que la forme de l'engagement individuel n'est jamais complètement prédéterminée par le collectif. Dans chacun des groupes étudiés, les modalités d'engagement varient d'un individu à l'autre, en fonction de son histoire personnelle, familiale, de son sujet de recherche, de sa conception de l'engagement. 
69 Ainsi, dans les trois cas, certains membres militent dans des mouvements de luttes urbaines, d'autres encore se sont tournés vers la politique et ont même été élus. C'est le cas d'un des fondateurs d'INURA, Richard Wolff, conseiller municipal à Zurich depuis 2013.

70 On notera que dans les trois cas, la politisation et la contestation étudiantes apparaissent souvent comme un des moteurs de l'engagement et de la volonté de créer de nouvelles solidarités de classe entre diplômés et populations plus marginalisées.

\section{Conclusion}

71 L'analyse de la géographie critique urbaine par les groupes qui s'en réclament donne un éclairage nouveau à l'analyse de ce courant de pensée. La géographie critique urbaine n'existe, comme tout autre courant de pensée, que parce que des communautés s'en réclament et cherchent à la diffuser en créant des supports et des activités.

72 La comparaison des trois groupes permet d'affirmer que, malgré les différences propres à chacun des contextes de production, la géographie critique urbaine présente bel et bien un socle commun: un ancrage normatif assumé, des référentiels théoriques communs (Harvey, Lefebvre, Smith, Soja), une grille d'analyse de la ville centrée sur les formes de domination et le souci de penser l'engagement et les conditions de production de la recherche.

73 Cela ne s'éloigne finalement pas réellement du positionnement de la géographie critique anglophone ou internationale telle qu'elle est présentée dans les revues (Antipode, IJURR, ACME...). Ces groupes de géographie critique ne sont donc pas en décalage, mais ils restent indéniablement peu visibles à l'échelle internationale, ce qui témoigne d'une géopolitique du savoir inégale.

74 Cette étude montre également que, dans les faits, les groupes qui se réclament explicitement de la géographie critique s'inscrivent dans un référentiel théorique essentiellement marxiste et post-marxiste. En ce sens, on pourrait dire qu'il s'agit de groupes de géographie critique ET radicale. Néanmoins, si le terme «critique » est le plus souvent mis en avant, c'est qu'il est finalement plus « accueillant » à la diversité des approches et des sensibilités des membres.

75 Au-delà de la question de l'ancrage théorique, ce qui apparaît dans l'analyse de cette "science en train de se faire", c'est le véritable souci réflexif et collectif du positionnement: quel engagement? Quelle modalité de production de la recherche? Quelle éthique de recherche? L'organisation en groupe de recherche auto-organisé permet de poser ces questions, même si cela ne garantit pas toujours de trouver des réponses définitives.

\section{BIBLIOGRAPHY}

En gras, les productions des groupes. 
ACME, An International E-Journal for Critical Geographies, 2015. Focus and scopes. [en ligne]. http://acme-journal.org/index.php/acme/about/editorialPolicies\#focusAndScope

B.A.S.S.D.A., 2006. A Kind of Queer Geography/Räume Durchqueeren: The Doreen Massey Reading Weekends. Gender, Place \& Culture: A Journal of Feminist Geography, 13(2), p. 173-186.

Berg L.-D., 2010. Critical Human Geography. In B. Warf (dir.), The Encyclopedia of Geography. Ed. Sage Publishers, vol. 2, p. 616-621.

Belina B., Best U. et Naumann M., 2009. Critical geography in Germany: from exclusion to inclusion via internationalization. Social geography, vol. 4, p. 47-58.

Belina B., Naumann M., Strüver A. (dir.), 2014. Handbuch Kritische Stadtgeographie. Westfälisches Dampfboot, Münster, 253 p.

Best U., 2009. The invented periphery: Constructing Europe in debates about "Anglo hegemony" in geography. Social Geography, p. 83-91. http://www.soc-geogr.net/4/83/2009/ sg-4-83-2009.pdf

Bourdieu P., 2001. Science de la science et réflexivité : cours du College de France 2000-2001. Paris, Raison d'agir, $237 \mathrm{p}$.

Calberac Y., Morange M., 2011. Appel à articles. Carnets de géographes.

Calberac Y., Morange M., 2012 Géographies critiques 'à la française'. Carnets de géographes, $\mathrm{n}^{\circ} 4$. http://www.carnetsdegeographes.org/carnets_debats/debat_04_01_Morange_Calberac.php

Carlos A. F. A. (dir.), 2006. Geografia na sala de aula. São Paulo, $8^{\mathrm{e}}$ éd., (Contexto, $6^{\mathrm{e}}$ éd.), $144 \mathrm{p}$.

Carlos A. F. A. 2012. Contra o produtivismo, um protesto solitário. [En ligne], consulté le 16 mai 2014, http://gesp.fflch.usp.br/es/node/187

Carlos A. F. A. (dir.), 2015. Crise urbana. Sao Paulo, Contexto, 192 p.

Cássia Ariza da Cruz R., 2012. Universidade Classe Mundial: paradoxos de um pensamento ao mesmo tempo neoliberal e neocolonialista. [En ligne], consulté le 16 mai 2014, http:// gesp.fflch.usp.br/node/181

Clerval A., 2010. Les dynamiques spatiales de la gentrification à Paris. Cybergeo : European Journal of Geography [En ligne], Espace, Société, Territoire, document 505, mis en ligne le 20 juillet 2010, consulté le 19 avril 2015. http://cybergeo.revues.org/23231; DOI : 10.4000/cybergeo.23231.

Foucault M., 1994 [1984]. Des espaces autres. Dits et écrits, 1954-1988, t. IV (1980-1988). Paris, Gallimard, p. 752-762.

Foucault M., 2001 [1984]. Qu'est-ce que les Lumières ? Dits et écrits, 1954-1988, t. II (1976-1988). Paris, Gallimard, p. 1381-1397.

Füller H., Michel B., 2014. 'Stop Being a Tourist!' New Dynamics of Urban Tourism in Berlin-Kreuzberg. International Journal of Urban and Regional Research, 38, p. 1304-1318.

Garnier J.-P., 2010. Une violence éminemment contemporaine. Essais sur la ville, la petite-bourgeoisie intellectuelle et l'effacement des classes populaires. Marseille, Agone, $256 \mathrm{p}$.

GESP. Apresentação. [En ligne], consulté le 16 mai 2014. http://gesp.fflch.usp.br/node/83

Gintrac C., Giroud M. (dir.), 2014. Villes contestées. Pour une géographie critique de l'urbain. Paris, Les Prairies ordinaires. 
Granjon F., 2015. Des fondements matérialistes de la critique. Revue française des sciences de l'information et de la communication [En ligne], 6 | 2015, mis en ligne le 23 janvier 2015, consulté le 06 septembre 2015. http://rfsic.revues.org/1257

Haas P. M., 1992. Epistemic Communities and International Policy Coordination. International Organization, 46/1, p. 1-35.

Harvey D., 2010. Géographie et capital, Vers un matérialisme historico-géographique. Paris, Syllepse, $282 \mathrm{p}$.

Harvey D., 2010. Le Nouvel Impérialisme, Paris, Les prairies ordinaires, 258 p.

Harvey D., 2012. Paris, capitale de la modernité. Paris, Les Prairies Ordinaires, 550 p.

INURA, 1991. INURA Bulletin, $n^{\circ} 1$, juin.

INURA, 1999. Possible urban worlds: urban strategies at the end of the 20th century. BirkhäuserVerlag, Basel/Boston/Berlin.

INURA, 2003. An alternative urban world is possible. A declaration for urban resaerch and action. Consulté le 14 août 2015. http://www.inura.org/v2/index.php/inura/declaration/.

INURA, 2004. The contested metropolis: six cities at the beginning of the 21st century. Birkhauser, Basel, Boston and Berlin.

Keucheyan R., 2013. Sociologie des pensées critiques contemporaines. In Fabien Granjon (dir.), De quoi la critique est-elle le nom ? Paris, Mare \& Martin, p. 95-111.

Kritische Geographie Berlin, 2012. Über uns, [En ligne], mis en ligne en 2012, consulté le 30 octobre 2014. http://kritische-geographie-berlin.de/?page_id=34

Kritische Geographie Berlin, 2014. Touristification in Berlin : Ein Bericht zur WorkshopReihe des Vereins Kritische Geographie Berlin. sub|urban. zeitschrift für kritische stadtforschung, vol. $2, \mathrm{n}^{\circ} 1, \mathrm{p} .167-180$.

Lamy E., Shinn T., 2006. L'autonomie scientifique face à la mercantilisation. Formes d'engagement entrepreneurial des chercheurs en France. Actes de la recherche en sciences sociales, 4/2006 (no 164), p. 23-50. www.cairn.info/revue-actes-de-la-recherche-en-sciencessociales-2006-4-page-23.htm

Latour B., 2007, [première édition 2001]. L'espoir de Pandore. Pour une version réaliste de l'activité scientifique. Paris, La Découverte.

Latour B., Woolgar S., 1979. Laboratory Life: the Social Construction of Scientific Facts. Los Angeles, Londres, Sage, $260 \mathrm{p}$.

Lefebvre H., 1968. Le Droit à La Ville, Paris, Editions Anthropos.

Lefebvre H., 2000 [1974]. La Production De l'Espace. Paris, Éditions Anthropos.

Meyer M., Moligneux-Hodgson S., 2011. «Communautés épistémiques » : une notion utile pour théoriser les collectifs en sciences? Terrains et travaux, 2011/1 ( $\left.{ }^{\circ} 18\right)$, p. 141-154.

Pestre D., 2005. Introduction aux Science Studies. Paris, La Découverte,

Pinto L., 2011. « « Neutralité axiologique », science et engagement. Une lettre de Pierre Bourdieu. Savoir/Agir, 2/2011 ( $\left.\mathrm{n}^{\circ} 16\right)$, p. 109-113.

Rodrigues A. M., 1988. Moradia nas Cidades Brasileiras. São Paulo, Editora Contexto.

Smith N., 2000. Who rules this Sausage Factory? Antipode, vol. 32, n 3, p. 330-339. 
Terray E. 2012. Penser à droite, Paris, Galilée.

\section{NOTES}

1. «La ville : la géographie dans la salle de classe»

2. «Le logement dans les villes brésiliennes"

3. An Alternative urban world is possible

4. LfbA-Lehrkräften für besondere Aufgaben

\section{INDEX}

Mots-clés: géographie critique, géographie radicale, études urbaines critiques, épistémologie de la géographie, science studies, production de l'espace urbain.

\section{AUTHOR}

\section{CÉCILE GINTRAC}

Cecile Gintrac, cecile.gintrac@gmail.com, est enseignante en classes préparatoires littéraires et membre du Laboratoire Mosaïques LAVUE UMR 7218 CNRS. Elle a publié récemment :

- Gintrac C., Giroud M. (dir.), 2014. Villes Contestées : pour une géographie critique de l'urbain. Les Prairies Ordinaires, Paris, $416 \mathrm{p}$. 